\title{
SHRINKING LUNG SYNDROME IN OVERLAPPING SYSTEMIC LUPUS ERYTHEMATOSUS AND SYSTEMIC SCLEROSIS: A CASE REPORT
}

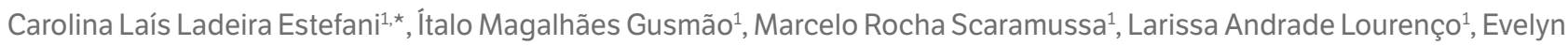

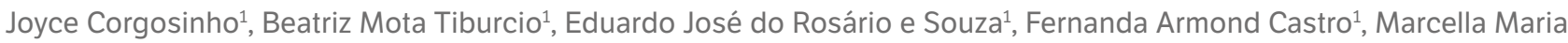
Soares Mello ${ }^{1}$

1.Santa Casa de Misericórdia de Belo Horizonte, Belo Horizonte (MG), Brazil.

*Corresponding author: carolinalestefani@hotmail.com

\section{BACKGROUND}

The shrinking lung syndrome (SLS) was first described in 1965 by Hoffbrand and Beck. It is a lung disease characterized by dyspnea, pleuritic chest pain, decreased lung volumes in the absence of pleuroparenchymal changes, restrictive functional pattern on pulmonary function tests, and elevation of the diaphragm. The SLS is a rare complication in patients with systemic lupus erythematosus (SLE) and has also been described in other multisystemic inflammatory diseases, such as systemic sclerosis (SS).

\section{CASE REPORT}

A 46-year-old woman, diagnosed with SLE in 2013, maintained regular follow-up with a specialist until 2016, when she used prednisone and hydroxychloroquine. In 2019, she returned to the specialist after evolving with sclerodactyly, microstomia, Raynaud's phenomenon, and esophageal dysmotility, when the diagnosis of limited cutaneous form SS was made in overlap with SLE. In 2020, she started presenting with dyspnea on minimal effort and ventilator-dependent chest pain of subacute onset. Additional propaedeutics was performed with spirometry, which showed severe restrictive disorder, with forced expiratory vital capacity at $40 \%$ of predicted, and simple chest radiography, with elevation of the diaphragmatic hemicups and decreased lung volume, especially to the right. The patient underwent chest computed tomography angiography, which showed no vascular or parenchymal alterations. She was then diagnosed with SLS.

\section{CONCLUSION}

Shrinking lung syndrome is a rare pulmonary complication, classically described in patients with SLE, and rarely in those with other multisystemic inflammatory diseases. This complication should be considered in those patients presenting with compatible symptomatology, typical radiological changes and restrictive pattern on pulmonary function tests, when other pulmonary complications more common in SLE and SS are excluded.

\section{KEYWORDS}

Systemic sclerosis, Shrinking lung syndrome, Systemic lupus erythematosus, Overlap syndrome. 